\title{
¿Almacenar o construir memoria? La educación patrimonial y el pensamiento crítico. ${ }^{1}$
}

por Belén M. Castro Fernández y Ramón V. López Facal

Universidad de Santiago de Compostela, España

belen.castro@usc.es,ramon.facal@usc.es

Recibido: 31/05/2017 - Aceptado: 12/06/2017

\section{Resumen}

El patrimonio cultural es una construcción intelectual que está mediada por la presencia de un discurso interpretativo. El establecimiento de una mirada crítica sobre el patrimonio o el proceso de patrimonialización por parte de la sociedad es fundamental. La asignación de valores a lo cotidiano, lo cercano y lo significativo está ligada con la mediación científica y la experiencia emocional del espectador. El patrimonio no es suficiente por sí mismo. Incluso si se pretende una experiencia únicamente estética y sensorial, es necesario manejar previamente referencias que permitan realizar este tipo de análisis. El conocimiento y la comprensión del patrimonio desencadenan la apropiación simbólica, la caracterización de contextos culturales y la definición de un referente identitario propio con valores específicos del presente. La capacidad de la educación para transformar el sentido de los elementos patrimoniales y ofrecer claves interpretativas es el punto de salida, o de llegada, de un juego mucho más amplio: la sostenibilidad como resultado de aprendizaje.

\section{Palabras claves}

educación patrimonial, patrimonio cultural, identidad, memoria

$$
* * *
$$

\section{Store or build memory? Heritage education and critical thinking.}

\section{Abstract}

Cultural heritage is an intelectual construction which is mediated by the presence of an interpretive discourse. The establishment of a critical look at the heritage or the patrimonialization society process is essential. The assignment of values to the everyday, the near and the significant is bound up with scientific mediation and the emotional experience of the viewer. Heritage is not enough by itself. Even if you intend to only aesthetic and sensory experience, it is necessary to previously handle references that allow you to perform this type of analysis. Knowledge and understanding of the heritage triggers the symbolic appropriation, the characterization of cultural contexts and the definition of a reference own identity with specific values of the present. The ability of education to transform the meaning of the assets and provide interpretative key is the point of departure or arrival of a much larger game: sustainability as a result of learning.

Keywords

heritage education, cultural heritage, identity, memory

Clío \& Asociados. La historia enseñada. 2017 (24) ISSN 2362-3063 (digital), pp. 51-58 Universidad Nacional del Litoral - Universidad Nacional de La Plata (Santa Fe/La Plata -Argentina) 


\section{Introducción}

Generalmente se asume que el patrimonio cultural es signo de identidad. Han sido muchos los cambios ocurridos en torno a su categorización, protección jurídica, conservación, interpretación y difusión. Sus límites se han ampliado más allá de las obras de arte, monumentos, conjuntos históricos y sitios arqueológicos. Por patrimonio cultural se entiende bienes y comportamientos que abarcan desde sitios naturales y tradiciones orales, pasando por rituales y artes del espectáculo, hasta un sinfín de elementos y manifestaciones tangibles o intangibles, entre las que se hallan incluso dietas gastronómicas como la mediterránea, declarada Patrimonio Cultural Inmaterial de la Humanidad por la UNESCO en el año 2010.

Algo está cambiando, tanto en las nuevas relaciones que han surgido entre patrimonio y cultura como en la sensibilidad de la sociedad, que reivindica la recuperación de las costumbres que la identifican y diferencian para convertirlas en elementos culturales. Esta nueva sensibilidad ha hecho posible que el patrimonio adquiera una dimensión más antropológica, dando paso a la transformación del patrimonio cultural en una cultura patrimonialista. Actitud que en tiempos recientes se ha convertido en una de las tablas de salvación para muchos lugares, que han visto emerger su economía y/o atractivo turístico gracias a la promoción y al uso realizados del patrimonio existente.

En las últimas décadas la toma de conciencia sobre el papel del patrimonio en la sociedad se presupone inherente al proceso de su puesta en valor. Desde el año 2005 esta concepción cobra mayor fuerza. Es entonces cuando el Consejo de Europa organiza en la ciudad de Faro la Convention on the Value of Cultural Heritage for Society.

Entre los acuerdos adoptados figura el compromiso de desarrollar la enseñanza del patrimonio cultural en todos los niveles de educación. Reforzar la cohesión social, mediante el fomento del sentido de responsabilidad compartida de la acción ciudadana. Promover la cualificación profesional de quienes participan en la gestión sostenible del patrimonio. Aprovechar al máximo el potencial económico de éste respetando sus valores. Fomentar el papel de iniciativas voluntarias interesadas en su conservación, como complemento a las funciones de las autoridades públicas. Incentivar el acceso y la participación democrática al patrimonio. Así como desarrollar el uso de las nuevas tecnologías en la difusión de sus contenidos.

A través de sus documentos, el Consejo insiste en iniciativas para tomar conciencia del valor y de la pluralidad de formas en que el patrimonio se manifiesta a lo largo de la historia. Entre otras, destacan los programas educativos, impartidos dentro y fuera del aula, con el objetivo de fomentar el conocimiento y la comprensión del patrimonio cultural. En este contexto, España, segundo país en bienes declarados patrimonio de la Humanidad, está poniendo en marcha un Plan Nacional de Educación y Patrimonio desde 2013, para desarrollar acciones formativas orientadas al aprendizaje de contenidos relacionados con el patrimonio, su puesta en valor y disfrute social.

Durante mucho tiempo, se ha tenido la falsa idea de que invertir en la conservación del patrimonio suponía un gasto extraordinario y excesivo que jamás podía verse rentabilizado. Sin embargo, se ha demostrado que una buena gestión del patrimonio implica que éste sea rentable no sólo desde el punto de vista social y cultural, sino también económico (Moreno, 1997).

Los valores económicos y sociales del patrimonio vienen fundamentados por su dimensión artística, histórica y de memoria, pero también por su capacidad de crear nuevos servicios que resulten productivos y de ofrecer diferentes canales de información que estimulen el mejor conocimiento de los elementos culturales que existen a nuestro alrededor (Hernández, 2002). Este uso del patrimonio al servicio de la ciudadanía ha de enmarcarse dentro de una dinámica de desarrollo sostenible, que compagine la correcta interpretación con una oferta cultural de calidad, contribuyendo a su protección y conservación.

Por eso, en los últimos tiempos se viene insistiendo en la creación de políticas culturales que favorezcan la gestión del patrimonio, como elemento clave del desarrollo social y económico. Todo ello con el máximo respeto hacia la diversidad cultural. En este proceso la educación formal, no formal e informal tiene un papel fundamental. A través de ella se puede afirmar que la dimensión 
cultural es un aspecto importante de la vida de las personas, que éstas han de integrarla de forma positiva y que ha de provocar una sensibilidad capaz de crear nuevos hábitos de conocimiento y uso del patrimonio. Esta actitud debiera resultar de la transmisión científica, crítica e histórica que proporciona la educación patrimonial. Con ella se puede contribuir a fomentar el respeto y la valoración social, que ha de estar en la base de cualquier actuación relativa al patrimonio cultural.

\section{El concepto de patrimonio a revisión}

Todo lugar de memoria posee dos niveles de lectura, como objeto conceptual y como objeto real. A nivel teórico, Maurice Halbwachs (1992) ya decía que la noción de memoria aplicada a la nación, a la colectividad y a la sociedad se había convertido en algo obvio. Los lugares del pasado son osarios de tradiciones, parques arqueológicos de la nostalgia conservadora y fuentes de relatos legendarios (Flor, 1998). Normalmente la dimensión memorial es reivindicada y manipulada a través de la gestión turística.

La polarización turística que sufren muchas ciudades históricas no sólo incide en la sobrecarga de los monumentos, también en la banalización de su espacio público y patrimonio. Su conservación no se destina en todos los casos a reconstruir una versión de la identidad para consumo local, sino turístico. Los turistas en vez de percepciones y experiencias auténticas, contemplan una tradición escenificada y ambientaciones forzadas. Una etnicidad reconstruida que convierte la escena urbana en producto (Calle, 2002).

Desde la Carta de Turismo Cultural adoptada por ICOMOS en 1976 se insiste en que la puesta en valor del patrimonio ha de ser una práctica interpretativa en vez de un ejercicio técnico e instrumental (Criado y González, 1996). De esta recomendación deriva la necesidad de desarrollar un proceso valorativo del patrimonio que permita reconstruir su uso actual. Se trata de escoger elementos patrimoniales como materia prima y reintroducirlos en un contexto actual donde adquiere valores nuevos. Ahí es donde debe entrar en escena la educación patrimonial, para que los agentes implicados realicen una correcta gestión educativa del patrimonio.

Para conseguirlo hay que partir del concepto actual de patrimonio, ampliamente revisado en las dos últimas décadas. En el ámbito científico parece estar superada la visión de patrimonio desde una perspectiva histórico-artística y monumental, pero en la sociedad esta concepción aún sigue siendo una constante. La introducción del concepto de patrimonio cultural en los años ochenta, fue un avance para la superación de esta acepción, sin embargo, a día de hoy vuelve a resultar incompleta ya que implica, en la mayor parte de las veces, la exclusión del patrimonio natural. De hecho, esta es la visión que se mantiene en la legislación educativa y en la mayor parte de los ámbitos sociales; lo natural comienza a ser tenido en cuenta a partir del concepto de paisaje cultural, en el que la naturaleza y la obra humana comienzan a ser indisociables (López, 2014).

En los últimos años, el creciente interés y la recuperación de los paisajes como elemento fundamental del patrimonio cultural marca nuevas formas de explotación, promoción y gestión del patrimonio en el marco de la planificación territorial (Dury, 2002; Fairclough y Rippon, 2002; Ermischer, 2002; Guido, 1999). Esta es una de las prioridades de las organizaciones europeas e internacionales. Desde 1992, la Convention concerning the Protection of World Cultural and Natural Heritage incluye los paisajes culturales como categoría de declaración. El European Landscape Convention, promovido por el Consejo de Europa en el 2000, considera al paisaje como un elemento clave del bienestar social. En este sentido algunos países han adoptado medidas para la protección y gestión de los paisajes culturales, como el English Heritage Historic Landscape Characterisation Programme o el Dutch Belvedere Programme.

Al amparo del programa Cultura 2000 de la Comisión Europea, se crea Pathways to Cultural Landscapes, una asociación de instituciones públicas y privadas que atiende a los paisajes culturales de toda Europa, a través de la investigación científica, la comunicación y la gestión sostenible. Lo más interesante de su contribución es que se dedica a regiones fronterizas, a paisajes marginales y a aquellos cuya imagen es la pobreza y la insignificancia histórica. Paisajes que a menudo no son percibidos como tales, pero que son la base y la expresión del patrimonio y la diversidad cultural. A

Clín \& Asaciados. La historia enseñada. 2017 (24) ISSN 2362-3063 (digital), pp. 5l-58. UNL - UNLP 
pesar de las particularidades de cada uno, todos ellos tienen una cosa en común, la vulnerabilidad frente a la sociedad de hoy. Los proyectos realizados por esta red ayudan a crear conciencia de las necesidades de estos paisajes, fomentan el sentido de pertenencia de la población local (rutas culturales, centros de información, formación de guías, intercambios escolares, etc.) y ayudan a establecer planes de desarrollo sostenible.

La noción de patrimonio avanza cada vez más hacia un enfoque integral. En este sentido, cabe destacar las aportaciones en las que se amplía y reformula el concepto añadiendo un elemento clave: las personas (Fontal, 2003). Existe una relación clara entre patrimonio y personas, que se puede conformar en torno al conocimiento, comprensión, respeto, valoración, disfrute y transmisión (Fontal, 2007). Bajo esta óptica, el patrimonio no son lugares, cosas u objetos, sino los vínculos que las personas establecen con lugares, cosas, recuerdos, etc. Sin personas non hay patrimonio, pues son quienes hacen que algo sea considerado o no patrimonio, mediante el proceso de patrimonialización. El factor humano es fundamental porque aporta identidad e introduce el debate sobre la contemporaneidad del patrimonio.

\section{La educación patrimonial: una perspectiva crítica}

Partiendo del Documento de Nara sobre la autenticidad (1994) todas las culturas y sociedades están arraigadas en formas particulares y en medios de expresión tangible e intangible, que constituyen su herencia y deben respetarse. Materiales y diseños, usos y funciones, tradiciones y técnicas, localización y contexto, espíritu y sentimientos, son factores que han de valorarse en la gestión y conservación del patrimonio para evitar la exclusión de minorías. Los principales problemas que afectan al patrimonio en general, y al inmaterial en particular, son los procesos de globalización y la aceleración de las transformaciones sociales, pues suponen riesgos de deterioro, desaparición y destrucción de su autenticidad (ICOMOS, 2011).

La educación patrimonial comunitaria, que implica a toda la sociedad, puede resultar peligrosa para los poderes que hacen uso del patrimonio en términos económicos. Incluso incómoda para contextos donde el patrimonio encarna mensajes ideológicos ya caducados. La educación patrimonial es necesaria para que las personas, en base a sus emociones y conocimiento, se identifiquen críticamente con el patrimonio, para que se abra el proceso selectivo de su identidad y para que las comunidades se auto-organicen en la custodia de su memoria. No se trata de mitificar los hitos, como tantas veces se viene realizando en manos del turismo. Tampoco primar la contundencia de los hitos arquitectónicos, su popularidad y monumentalidad visible, que oculta el valor del resto del conjunto. Sino de revisar el paisaje cultural como espacio de almacenamiento de la memoria y reconocer en él valores para la convivencia ciudadana.

Algo es patrimonio cultural porque alguien le otorga un valor representativo de su comunidad, más allá, en muchas ocasiones de lo legalmente protegido; por tanto, es una construcción social (López, 2014). Lo patrimonial es una cualidad añadida por las personas y por tanto sujeta a los cambios en las percepciones y el comportamiento humano (Cuenca, 2013). Esto conduce a procesos de despatrimonialización (Llonch, 2015) y a cuestionar el discurso oficial.

Está claro que el concepto de patrimonio tiene un carácter polisémico, inacabado y complejo de definir. Una visión completa es la concepción holística y simbólico-identitaria (Cuenca, Estepa y Martín, 2011), a partir de la que se concibe la educación patrimonial como una disciplina que lleva a construir valores de identidad, fomentar el respeto intercultural y el cambio social. Formar una ciudadanía socioculturalmente comprometida. Entre sus objetivos estarían los propiamente educativos, los que revierten en los elementos patrimoniales -conservar y proteger-, y otros como el desarrollo territorial o la socialización del patrimonio (Cuenca, 2013).

La educación patrimonial comienza a adquirir importancia a finales de la década de los noventa. Desde entonces se tiene avanzado considerablemente en el diseño de sus componentes: objetivos, contenidos y metodología. La educación patrimonial no constituye un fin en sí mismo, sino que debe integrarse en las metas establecidas para la educación reglada, especialmente en la formación de la ciudadanía, pues desde una perspectiva crítica asume toda una serie de finalidades: procesos 
de identificación (individual, colectiva y supra-colectiva), comprensión de las sociedades pasadas y presentes, defensa y protección de los elementos patrimoniales, etc. En realidad, no debiera existir una materia específica sobre educación patrimonial, sino que su enseñanza habría que incorporarlo al resto de asignaturas. En cuanto a los contenidos, es necesario que sean coherentes con los propósitos que se persiguen pudiendo ser conceptuales, procedimentales y actitudinales. Adquirir claves interpretativas para poder aplicarlas al presente. Y metodológicamente el eje fundamental sería partir del entorno y de problemas e intereses próximos al alumnado, recogiendo información de diversas fuentes hasta llegar a conclusiones sobre los problemas formulados inicialmente (Cuenca, Estepa y Martín, 2011). En suma, con la educación se incide en la selección patrimonial.

En los modelos educativos patrimoniales existentes es interesante destacar el diseño de la sensibilización como estrategia de enorme potencialidad. El esquema a seguir es el siguiente: conocer para comprender, comprender para respetar, respetar para valorar, valorar para cuidar, cuidar para disfrutar, disfrutar para transmitir y transmitir para conocer (Fontal, 2007). La valoración es el elemento clave, el indicador de sensibilización.

Las posibilidades de abordar el patrimonio en la didáctica de las ciencias sociales son muy amplias. Además de la adquisición de competencias y contenidos (Calaf y Marín, 2015), los procesos de identificación que provoca favorecen la creación y el refuerzo de los vínculos identitarios entre las personas y su entorno. Esto es clave para fomentar el desarrollo socioeconómico del territorio. Desde que en los años setenta Hugues de Varine redefine el museo ampliando su dimensión de edificio a región, de colección a patrimonio regional, de público a comunidad regional participativa, el triángulo territorio-patrimonio-comunidad se establece como el fundamento de la nueva museología. Para integrar a la población en su acción, se apuesta por nuevos métodos de comunicación y de gestión basados esencialmente en reflejar la identidad de los pueblos y asociarla a proyectos de futuro. Estos ecomuseos, o museos comunitarios como también se les conoce, llevan funcionando desde entonces con más o menos acierto. Saber extraer las potencialidades importantes de la identidad cultural, saber interpretarlas y difundirlas en los nuevos museos o centros de interpretación implica, el éxito o el fracaso de una iniciativa cultural. Por eso cada vez es más importante recurrir a fórmulas con enfoque creativos y particulares que desde la educación patrimonial permitan revisar la práctica museológica, y así adaptarla a las limitaciones y posibilidades de cada territorio.

Esta mirada renovadora también puede ser aplicada a los itinerarios culturales, que se presentan como una alternativa válida a la preservación patrimonial. Más allá de su vinculación con rutas tradicionales, pueden ser vistos con una significación mayor. Así como la noción de monumento se ha ido ampliando, de obra aislada o singular a la conjunción de ésta con su entorno, los itinerarios pueden conferir a la preservación del patrimonio una amplitud territorial y una integración cultural (García, 2002). Basta revisar a autores como Eleonora Berti (2012) para comprobar investigaciones en el campo del paisaje y la identidad, dentro del programa de los Itinerarios Culturales creado por el Consejo de Europa. Con todo, y como ya apuntaba Anthony Giddens, la globalización impulsa el resurgimiento de identidades culturales locales, de modo que este movimiento de ida y vuelta de la escala de lo global a lo local, permite revisar las oportunidades de los itinerarios como estrategias de conocimiento y puesta en valor. Al mismo tiempo, los itinerarios permiten trabajar con las influencias recíprocas, entre los lugares centro y los llamados periféricos, en tiempos de gran conectividad.

\section{La sostenibilidad como resultado de aprendizaje}

Decía Paulo Freire (2003:34) que en la medida en que nos tornamos capaces de intervenir, capaces de cambiar el mundo, de transformarlo, de hacerlo más bello o más feo, nos volvemos seres éticos. Si una persona se sensibiliza con algo, porque previamente su emoción le ha despertado el interés de conocerlo y comprenderlo, está mucho más preparada para reivindicar su conservación. Esta actitud de defensa respecto al patrimonio debe enfocarse desde una perspectiva que promueva el desarrollo de valores éticos y cívicos para aprender no sólo a respetar nuestra herencia, sino

Clín \& Asaciados. La historia enseñada. 2017 (24) ISSN 2362-3063 (digital), pp. 5l-58. UNL - UNLP 
también la de los otros. Caminar desde el respeto por el patrimonio más personal hasta aquel más universal y ligado a nuestro presente histórico. La sensibilización a la hora de trabajar estos vínculos y conexiones parece el camino más adecuado para conseguir la implicación de las personas.

La educación en materia de comportamiento cívico y patrimonio cultural exige la participación social y la integración de campañas dentro y fuera de los sistemas educativos: acciones educativas en centros de enseñanza, acciones educativas formativas para madres y padres, acciones educativas para jóvenes dentro de un programa municipal de ocio alternativo saludable, así como la puesta en marcha de campañas de sensibilización. En esta línea de trabajo se desarrolla el Plan Rúas (Regeneración Urbana Arte Santiago). Un programa para la conservación de la zona histórica de Santiago de Compostela (A Coruña) libre de pintadas y para la recuperación de espacios mediante la intervención artística, que lleva a cabo la entidad Vella Escola especializada en actividades relacionadas con cultura urbana (Figura 1). Se trata de una iniciativa educativa en valores que apuesta por la sostenibilidad y la conservación preventiva como resultado de aprendizaje.

El graffiti en Santiago de Compostela es un fenómeno con clara presencia en la periferia de la ciudad (medianas, edificios ruinosos, túneles). En los últimos años ha ido creciendo y ganando presencia en la zona nueva metropolitana (persianas de negocios, señales, contenedores) llegando a afectar al casco histórico de la ciudad, en el que se han visto dañados espacios y monumentos de alto interés patrimonial. La complejidad y el elevado coste que suponen para las administraciones públicas y los particulares los tratamientos de limpieza y restauración de los bienes dañados, requieren la puesta en marcha de programas educativos destinados a este fin. La mayor parte de los ayuntamientos disponen de ordenanzas municipales de limpieza y en materia de sanciones, pero en pocas ocasiones se han desarrollado programas educativos consistentes, orientados a la formación e información de los jóvenes para la prevención de este tipo de conductas. Teniendo en cuenta la situación de Compostela, el ayuntamiento dispuso la necesidad de desarrollar el Plan Rúas orientado a la prevención, ya que las medidas sancionadoras adoptadas hasta el momento no resultaban lo suficientemente disuasorias como para evitar futuras intervenciones en este campo. El Plan se ha diseñado para ser implementado durante un año y medio en dos fases, la primera desde marzo de 2016 a marzo de 2017 y la segunda fase de marzo a septiembre de 2017.

Figura 1. Plan Rúas, Santiago de Compostela.
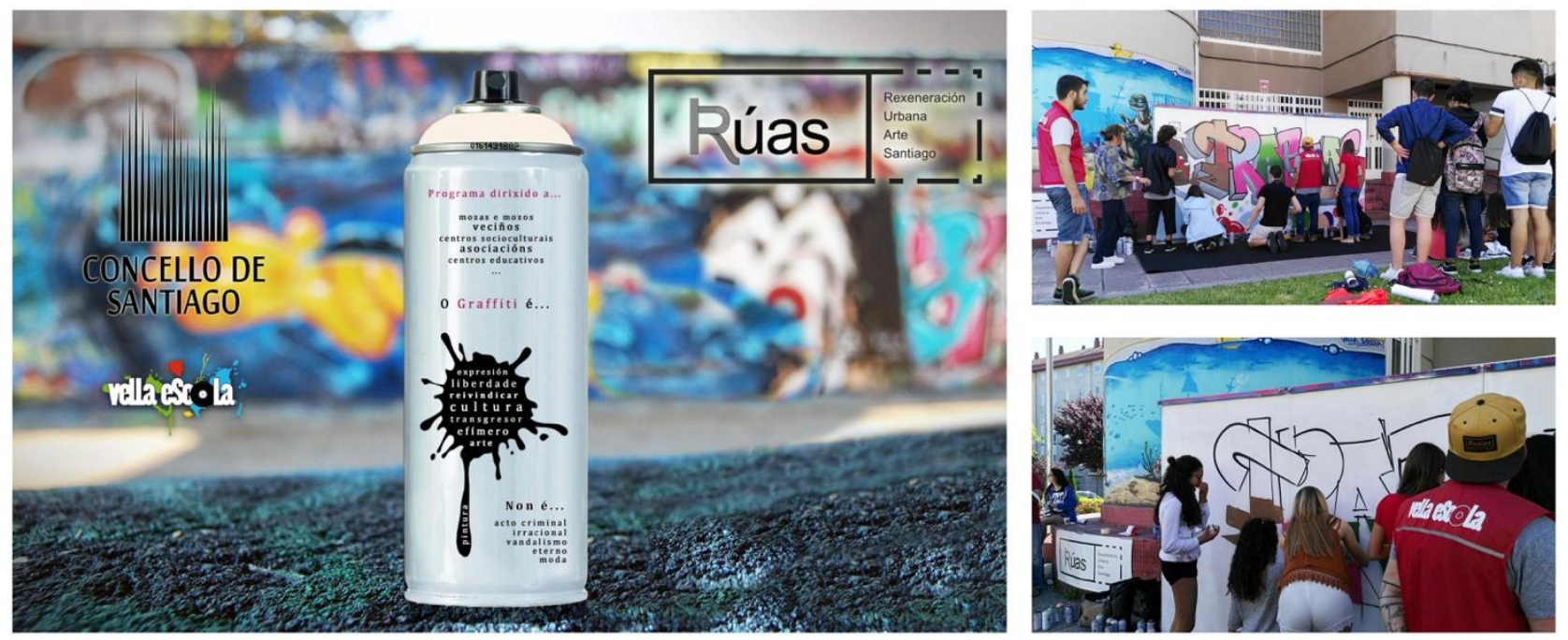

www.facebook.com/planRUAS/, 2017

Con este programa se persigue prevenir la aparición de nuevas pintadas y rescatar espacios públicos afectados, logrando beneficios visibles a corto plazo (recuperación de muros y fachadas) y a medio plazo (disminución de pintadas). La finalidad es mejorar la imagen de las calles de 
Santiago de Compostela y preservar el patrimonio histórico y cultural libre de pintadas, desarrollando un programa de prevención centrado en acciones informativas y actividades educativas dirigidas principalmente al colectivo juvenil de la ciudad.

La metodología pasa por consolidar la cultura de prevención del vandalismo y de las prácticas antisociales concernientes a las pintadas y al graffiti en la ciudad. Para ello se hace necesario brindar un espacio de expresión entre los miembros de la comunidad y los graffiteros, promoviendo el muralismo como expresión de arte urbano. De este modo, el mural aporta además otros valores como el refuerzo de la identidad de los barrios y el mensaje social positivo. También se impulsan medidas de concienciación social y ciudadana, como el fomento del graffiti autorizado en espacios abiertos, exhibiciones, talleres, festivales, etc.

La participación es el criterio de éxito y para conseguirlo la información es clave. Si la ciudadanía al completo es consciente de la gravedad de las pintadas, en coste económico y de imagen para la ciudad, estará más sensibilizada y dispuesta a colaborar y evitarlas. La sociedad en general debe descubrir el grafiti como una forma de expresión artística que, si cumple los requisitos legales, se puede disfrutar en la ciudad.

El Plan prioriza las actuaciones preventivas frente a las correctivas, haciendo partícipe a los diferentes colectivos de la ciudad (asociaciones juveniles, culturales, vecinales, sociales...) en la recuperación de los espacios afectados por las pintadas, a través de un proceso de cambio que suponga el paso de una ciudad llena de pintadas a una ciudad libre de ellas y referente turístico del arte urbano (muralismo) con pinturas que hablen de la ciudad y de la identidad de sus barrios. De hecho, prevé la restauración de zonas afectadas y muros en mal estado con obras murales que versen sobre la ciudad y sobre elementos que identifiquen el barrio donde se lleve a cabo la intervención artística. Con ello se buscar potenciar el sentimiento de pertenencia y conseguir una mayor implicación y participación social en el programa.

\section{Apuntes finales}

El patrimonio cultural nunca está aislado, hay un contexto y dentro de él las personas. Sin ellas no tiene sentido. Si su conservación depende en buena parte de la comprensión ciudadana, parece claro la importancia de aumentar el conocimiento sobre él. Dado que no hay déficit de reconocimiento de la educación en la legislación patrimonial y tampoco en la educativa, es necesario impulsar la educación patrimonial de manera efectiva en los ámbitos formal, no formal e informal, para que la sociedad pueda acceder al patrimonio con una mediación profesional. Este aprendizaje ha de mejorar su interpretación y valoración del patrimonio como recurso formativo, al tiempo que turístico. La educación patrimonial puede facilitar las herramientas necesarias y las claves interpretativas para dar respuesta al presente y emprender acciones de mejora. Contribuir, asimismo, al desarrollo del pensamiento crítico, la capacidad de selección, la construcción de la identidad y la identificación de valores. Y es que la educación patrimonial, valiéndose de los símbolos como instrumento de trabajo, es en sí misma conocimiento. Como recoge Llorenç Prats (1997: 13) de una inscripción en una pintura naif Aprende, si eres sensato, pues más vale ciencia que herencia.

\section{Bibliografía}

Berti, E. (2012). Itinerari culturali del Cansiglia d'Eurapa. Tra rícerca di identità e pragetto di paesaggio. Florencia: Firenze University Press.

Calaf, R. \& S. Marín (2012). Adalescencia y educación patrimonial. Aula de innavación educativa, 208, 18-21.

Calle Vaquero, M. (2002). La ciudad histórica coma destino turística. Barcelona: Ariel.

Criada Boada, F. \& C. A. González Pérez (1996). Fichas e redes, sitios e paisaxes: das bases de datas ós sistemas de infarmación, dos inventarios estáticas ús catálogus interactivos. En Fontenla San لuan, ¿. (courd.). Us profesianais da 
Histaria ante a Patrimanio Cultural: liñas metadalóxicas (ID3-IZI). Santiago de Campastela: Dirección Xeral de Patrimonio Cultural.

Cuenca López, J. M. (2003). Análisis de concepciones sobre la enseñanza del patrimanio en la educación obligataria. Enseñanza de las Liencias Saciales, 2, 37-45.

Cuenca López, J. M. (2013). El papel del patrimanio en los centros educativas: hacia la sacialización patrimanial. Tejuela: Didáctica de la Lengua y la Literatura. Educación, 19,76-㿟.

Cuenca López, J. M.; Estepa Jiménez, J. \& M. J. Martín Cáceres (2011). El patrimonio cultural en la educación reglada. Patrimanio Cultura/ de España, 5, 45-57.

Dury, P. (2002). The historic and cultural dimensions of landscape. Naturapa, 98, 12-13.

Ermischer, G. (2002). How to gain European understanding of the cultural landscape. Recuperado de: http://www.pcleu.de/project/virt_lib/understanding.pdf.

Fairclough, G. \& S. Rippon (ed.) (2002). Eurape's cultural lanscape: archeologists and the management of change. Brussels: Eurapa Archeolagiae Consilium.

Flor, F. R. (1998). Los lugares de la memoria: el intelectual y el aura de la ciudad histórica entre dos fines de siglo. En Castilla Oreja, M. A. (Ed.). Centras Históricas y canservación de/ patrimanio (127-141). Madrid: Fundación Argentaria.

Fontal Merillas, 0. (2003). La educación patrimanial. Definición de un madelo integral y diseño de sensibilización. Tesis dactaral. Ovieda: Universidad de Qviedo.

Fontal Merillas, D. (2007). El patrimanio cultural del entarno próxima: un diseño de sensibilización para secundaria. Enseñanza de las Liencias Saciales, 6, 3l-47.

Freire, P. (2003). El grita mansa. Buenos Aires: Sigla XXI.

García Miranda, R. (2002). Inventarios de itinerarios culturales en los tiempos de la globalización. En AA.V. El patrimunia intangible y atros aspectos relativas a los itinerarios culturales. Congreso Internacional del Comité Internaciona/ de Itinerarios Culturales (CIIC) de ICDMDS (I23-128). Navarra: Institución Príncipe de Viana.

Guido, M. R. (1999). Landscape: a natural and cultural Heritage. Naturapa, II, 15.

Halbwachs, M. \& A. C. Lewis (1992). On callective memory. Chicago: University of Chicago Press.

Hernández Hernández, F. (2002). El patrimania cultural: la memaria recuperada. Cijón: Trea.

ICDMDS (2001). El valar intangible del patrimanio. Recuperado de: http://www.esicamos.org/Nueva_carpeta/LIBRD__RITERIDS/SEVILLA.htm.

López Cruz, I. (2014). La Educación Patrimanial. Análisis de/ tratamienta didáctica de/ patrimanio en los libros de texto de CLSS en la Enseñanza Secundaria. Tesis doctoral. Huelva: Universidad de Huelva.

Llanch Molina, N. (2015). La educación patrimanial como herramienta de "rebeldía ciudadana". Educaçãa histórica e educaçãa patrimunial- novos desafíns. En Solé, G. (coord.). I/ Seminário Internacional de Educação Patrimanial: Cantributos para a construçãa de uma consciência patrimunia/ (37-52). Braga: Universidade do Minho, Instituto de Educação.

Moreno de Barreda, F. (1997). La dimensión ecanómica del patrimanio arquitectónica: punto de partida para soluciones nuevas. Patrimania Lultural y Derecha, I, 213-230.

Prats, LI. (1997). Antropología y patrimunio. Barcelona: Ariel.

\section{Notas}

${ }^{1}$ Agradecimientos. Este trabajo forma parte de la investigación financiada por el Plan Nacional de I+D+i del MINECO (EDU2015-65621-C3-1-R) y cofinanciado con fondos FEDER de la UE. 